\title{
Stability, Phase and Absorption Analysis of 4-Alkenyl Bicyclohexylnitrile: Thermodynamic and Spectroscopic Approaches
}

\author{
Rashmi Rekha Sahoo, ${ }^{1}$ K. Subrahmanya Sarma ${ }^{2}$ and P. Lakshmi Praveen ${ }^{{ }^{*}}$ \\ ${ }^{1}$ Department of Physics, Veer Surendra Sai University of Technology, \\ Burla-768018, Sambalpur, Odisha, India \\ ${ }^{2}$ Department of Physics, MVN, JS \& RVR College, Malikipuram-533253, \\ East Godavari, Andhra Pradesh, India \\ *Corresponding author: plpraveen_phy@vssut.ac.in
}

Published online: 25 April 2021

To cite this article: Sahoo, R. R., Sarma, K. S. \& Praveen, P. L. (2021). Stability, phase and absorption analysis of 4-alkenyl bicyclohexylnitrile: Thermodynamic and spectroscopic approaches. J. Phys. Sci., 32(1), 27-38. https://doi.org/10.21315/jps2021.32.1.3

To link to this article: https://doi.org/10.21315/jps2021.32.1.3

\begin{abstract}
Thermodynamic and spectroscopic approaches have been adopted to analyse the stability, phase and absorption analysis of 4-alkenyl bicyclohexylnitrile. The atomic net charge and dipole moment at each atomic centre have been evaluated using the complete neglect differential overlap (CNDO/2) method. The modified RayleighSchrodinger perturbation theory, along with multicentered-multipole expansion method, has been employed to evaluate long-range intermolecular interactions, while a "6-exp" potential function has been assumed for short-range interactions. The total interaction energy values of the alkenyl compound in tetrahydrofuran (THF) solvent have been calculated and compared with the values of the pure form of the compound. These energy values have been used as the input to calculate the thermodynamic parameters of each configuration at room $(300 \mathrm{~K})$ and nematic-isotropic transition $(364.7 \mathrm{~K})$. The UV-Visible spectra of the compound in pure form and THF medium have been studied. The phase and $U V$ stabilities of the compound have been discussed.
\end{abstract}

Keywords: 4-alkenyl bicyclohexylnitrile, stability, phase analysis, absorption, spectroscopic approach

\section{INTRODUCTION}

Liquid crystals belong to the class of soft condensed matter physics which deals with complex structural and dynamic properties intermediate between true liquids and crystalline solids. ${ }^{1,2}$ These are highly sensitive to external fields, boundary

(C) Penerbit Universiti Sains Malaysia, 2021. This work is licensed under the terms of the Creative Commons Attribution (CC BY) (http://creativecommons.org/licenses/by/4.0/). 
effects and thermal fluctuations..$^{3-5}$ This sensitivity imposes new interesting challenges to be tackled by the entire scientific community and thus widens the avenues of technological applications. To a less-trained person, the term liquid crystal appears quite paradoxical, suggesting the juxtaposition of two distinct liquid and solid states that have defined properties like the fluidity of the former and rigidity of the latter. Given this scenario and multi-disciplinary application of liquid crystals, it becomes both intriguing and exciting for the scientific intelligentsia. ${ }^{6,7}$

The present theoretical analysis aims at stability, phase and absorption analysis of a mesogen 4-alkenyl bicyclohexylnitrile based on thermodynamic and spectroscopic approach for understanding the structure stability relation and molecular interactions. ${ }^{8}{ }^{8}$ It is essential to determine the various factors contributing to the phase stability at a certain temperature. The most significant factor responsible for phase behaviour is the intermolecular forces operating either over a long or short range. ${ }^{10,11}$ If the molecules involved in the interaction are oppositely charged, they experience electrostatic forces of interaction over quite a long distance which might cause a strain in the bond lengths along the direction of attractive forces or disturb the arrangement of the assembly of molecules adversely disturbing the phase. If the molecules bear the same charge, they might experience repulsive forces only in the vicinity of small distances, resulting in small distortion in the geometry of the molecular arrangement. In addition to this, the difference in the atoms' electro-negativity leads to polarity and hence origin of dipole moment. Polarisation arises due to the difference in the electro-negativity of two atoms present either in the crystal or the solvent, which has been added to achieve a better view. While considering the electrostatic energy, all types of interaction starting from monopole-monopole, monopole-dipole and dipole-dipole terms are included. ${ }^{12}$

Thus, the energy associated with the molecular pair interaction is evaluated taking electrostatic, repulsive, polarisation and dispersion energy into account. The distance between two molecules during interaction should be an intermediate range as extreme proximity might result in van der Waals contact, and large distance would affect the inclusion of repulsive forces in interaction energy calculation. Liquid crystals consist of an assembly of molecules with comparatively larger degrees of freedom than true solids. Further, the study of the effect of change in macroscopic variables like temperature leads to a change in microscopic behaviour due to molecular interactions, resulting in a deviation in microscopic variables like entropy, internal energy and free energy. Thus, the statistical thermodynamic approach is well suited for the above objectives. ${ }^{13}$ 


\section{COMPUTATIONAL DETAILS}

\subsection{Computation of Interaction Energy and Thermodynamic Parameters}

The molecular geometry of alkenyl compound has been constructed based on the published crystallographic data with standard values of bond lengths and bond angles. ${ }^{14}$ In the present computation, the complete neglect differential overlap $(\mathrm{CNDO} / 2)$ method has been employed to compute the net atomic charge and dipole moment at each atomic centre of the molecule. ${ }^{15}$ A detailed computational scheme based on Claverie's simplified formula for evaluating interaction energy between a molecular pair has been used to calculate the energy for fixed configuration. ${ }^{16}$ According to the second-order perturbation theory for intermediate range interactions, the total pair interaction energy of molecules $\left(U_{\text {pair }}\right)$ is represented as a sum of various terms contributing to the total energy: ${ }^{17}$

$$
U_{\text {pair }}=U_{e l}+U_{p o l}+U_{\text {disp }}+U_{\text {rep }}
$$

where $U_{e l}, U_{p o l}, U_{\text {disp }}$ and $U_{\text {rep }}$ are the electrostatic, polarisation, dispersion and repulsion energy terms, respectively.

Again, electrostatic term is expressed as: $U_{e l}=U_{Q Q}+U_{Q M I}+U_{M I M I}+\ldots \ldots \ldots$

where $U_{Q Q}, U_{Q M I}$ and $U_{M I M I}$, etc., are monopole-monopole, monopole-dipole and dipole-dipole terms, respectively. ${ }^{17}$ The computation of electrostatic term has been restricted only up to dipole-dipole energy term. In the present computation, the dispersion and short-range repulsion terms are considered together because several semi-empirical approaches, viz. the Lennard-Jones or Buckingham type approach, actually proceed in this way. Kitaygorodsky introduced a Buckingham formula whose parameters were later modified by Kitaygorodsky and Mirskay for hydrocarbon molecules and several other molecules and finally gave the expression: ${ }^{18}$

$$
\begin{aligned}
& U_{\text {disp }}+U_{\text {rep }}=\sum_{\lambda}^{(1)} \sum_{\nu}^{(2)} U(\lambda, \nu) \\
& U(\lambda, \nu)=K_{\lambda} K_{\nu}\left(-A / Z^{6}+B e^{-\gamma Z}\right)
\end{aligned}
$$

where $Z=R_{\lambda v} / R_{\lambda v}^{0} ; R_{\lambda v}^{0}=\left[\left(2 R_{\lambda}^{w}\right)\left(2 R^{w}{ }_{v}\right)\right]^{1 / 2}$, in which $R_{\lambda}^{w}$ and $R^{w}{ }_{v}$ are the van der Waals radii of atom $\lambda$ and $v$, respectively. The parameters $A, B$ and $\gamma$ do not depend on the atomic species. But $R_{\lambda v}^{0}$ and factor $K_{\lambda} K_{v}$ allow the energy minimum to have different values according to the atomic species involved. The necessary formulae may be found elsewhere. ${ }^{19}$ An orthogonal coordinate system is considered to facilitate the above calculation. The origin on an atom has been chosen at almost 
midpoint of the molecule. The $\mathrm{x}$-axis along a bond parallel to the long molecular axis while the $y$-axis lies in the molecule plane and z-axis perpendicular to the molecular plane. The total interaction energy values obtained through these computations have been used as input to calculate the following thermodynamic parameters to explain order-disorder phenomenon at molecular level: ${ }^{20}$

$$
\begin{aligned}
& A=-k T \ln \Sigma_{i} \exp \left(-\beta \varepsilon_{i}\right) \\
& S=k \ln \Sigma_{i} \exp \left(-\beta \varepsilon_{i}\right)+(U / T) \\
& U=\Sigma_{i} \varepsilon_{i} \exp \left(-\beta \varepsilon_{i}\right) / \Sigma_{i} \exp \left(-\beta \varepsilon_{i}\right)
\end{aligned}
$$

where $A$ stands for Helmholtz free energy, $U$ stands for internal energy and $S$ stands for entropy. Meanwhile, $\beta=1 / k T, k$ is the Boltzmann constant, $T$ is the absolute temperature, and $\varepsilon_{i}$ represents the energy of the configuration $i$ to the minimum energy value.

\subsection{UV-Visible Absorption Spectra}

The configuration interaction (CI) method is widely used for the electronic absorption spectra calculation. CI method has been used coupled with a semiempirical model Hamiltonian for an absorption spectra of many LC and organic molecules. ${ }^{21,22}$ Hence, CNDO/S+CI method has been used for the calculation of electronic spectra. These results have been compared with the INDO/S + CI method. A revised version program of QCPE 174 has been used for this purpose. ${ }^{23}$

\section{RESULTS AND DISCUSSION}

The choice of distance during the two modes of interactions has been made to eliminate the possibility of van der Waals contact and to keep the molecule within the short- and medium-range interactions. Again, translations along $\mathrm{x}$-axis have been studied. The geometry of the alkenyl molecule has been constructed, and the transition temperature has been noted from the published crystallographic data. ${ }^{14}$ The structure of alkenyl compound has been shown in Figure 1. The total interaction energy values in the vacuum of an alkenyl compound have been reported. ${ }^{24}$ 


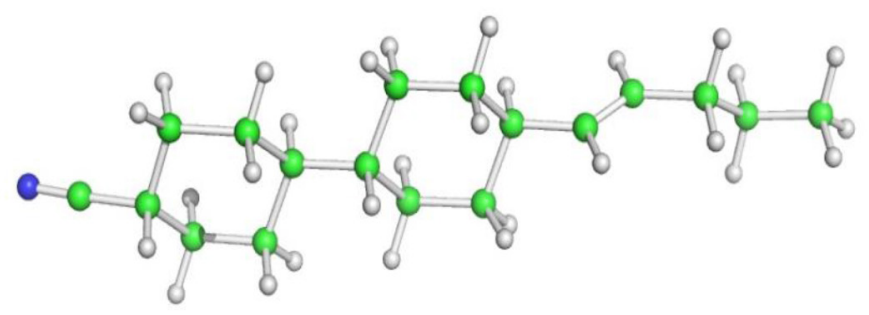

Figure 1: Structure of 4-alkenyl bicyclohexylnitrile.

\subsection{Stacking Interactions}

In a molecular pair, one of the interacting molecules is fixed in the $x-y$ plane, while the second has been kept at a separation of $6 \AA$ along the $\mathrm{z}$-axis with respect to the fixed one. The arrangement of the molecules during various modes of interactions has been shown elsewhere. ${ }^{25}$ The variation of total interaction energy of alkenyl compound in the THF solvent and energy in a vacuum with respect to a translation along $\mathrm{x}$-axis corresponding to a configuration $\mathrm{y}\left(0^{\circ}\right) \mathrm{z}\left(180^{\circ}\right)$ is shown in Figure 2. It is found that energy is constant over a range of $0 \pm 2 \mathrm{~A}^{\circ}$ (pure), $0 \pm 6 \mathrm{~A}^{\circ}$ (solvent). The data suggest that the sliding of one molecule over the other is energetically allowable and permissible within a very small region and thus contributes to ordering in mesogens as it can be linked to lower fluidity and greater ordering. The variation of interaction energy components with respect to rotation about the $\mathrm{x}$-axis corresponding to configuration $\mathrm{y}\left(0^{\circ}\right) \mathrm{z}\left(0^{\circ}\right)$ reveals that the dispersion energy is the principal component of total energy. The role of the polarisation component is minor and is almost consistent for the entire range. The electrostatic component magnitude is much lower to the dispersion component. The minimisation of energy has been carried out with respect to translation and rotation about all axes. The translational accurateness of $0.1 \AA$ and $1^{\circ}$ in the rotation of one molecule with respect to other has been accomplished. Generally, the path of energy optimisation firmly relies upon the computational objective. The global investigation for minimum energy configuration or the study of interaction energy variation under pre-decided proviso will have an entirely diverse path. Hence, one needs to be cautious in the selection of the specific route. 


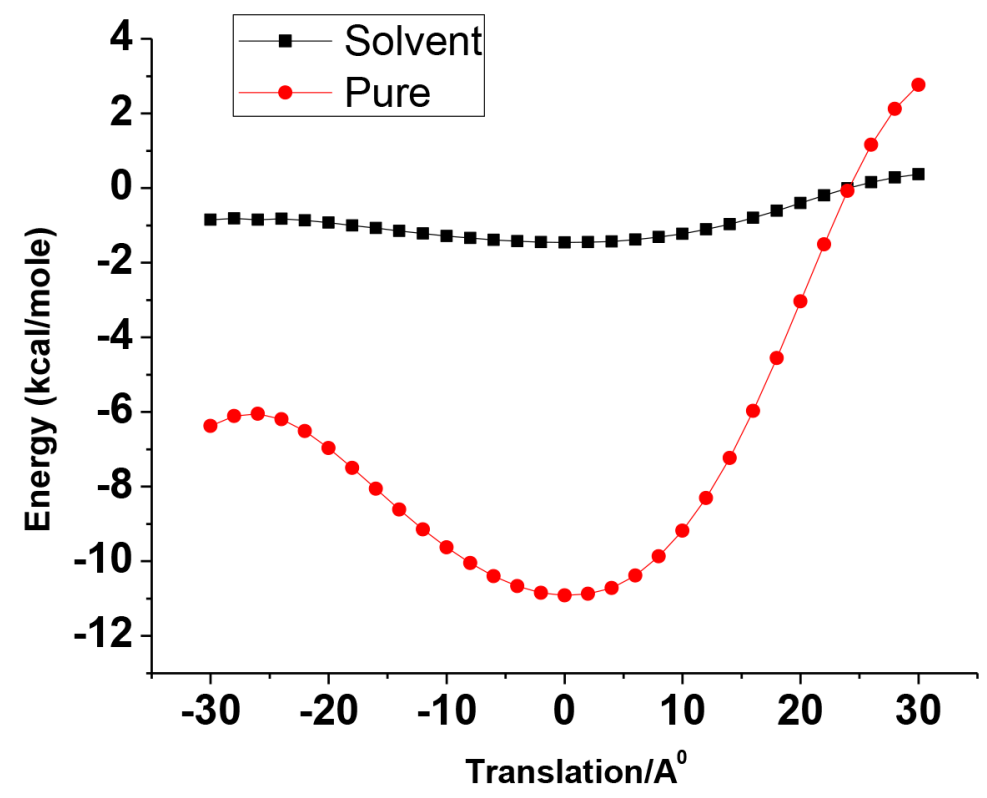

Figure 2: Variation of stacking interaction energy components with respect to translation along $\mathrm{x}$-axis corresponding to the configuration y $\left(0^{\circ}\right) \mathrm{z}\left(180^{\circ}\right)$ for pure alkenyl compound and in the solvent (THF).

\subsection{In-plane Interaction}

This is also called side to side interaction, where keeping one interacting molecule fixed along $\mathrm{x}$-axis the other molecule is kept along the $\mathrm{y}$-axis. The interacting molecule has been kept at a separation of $8 \AA$ along the $y$-axis with respect to the fixed one. ${ }^{25}$ Again, rotations about the $y$ - and x-axes have been given, and the corresponding energy has been minimised with respect to translation and rotation about all axes. Further similar computations are carried out, and the results yielded manifest the region over which energy remains constant and hence the degrees of freedom associated with this planar orientation. Considering the translation along the $\mathrm{x}$-axis for the configuration $\mathrm{y}\left(0^{\circ}\right)$ is shown in Figure 3 during the translational motion for the alkenyl compound in THF solvent and compared with the vacuum values. The data reflect that energy is nearly constant over a range of $10 \pm 6 \mathrm{~A}^{\circ}$ (solvent) and $4 \pm 2 \mathrm{~A}^{\circ}$ (pure). This constancy over a larger range suggests that it can maintain its positional ordering as a molecular pair to a greater extent against increased thermal agitation. 


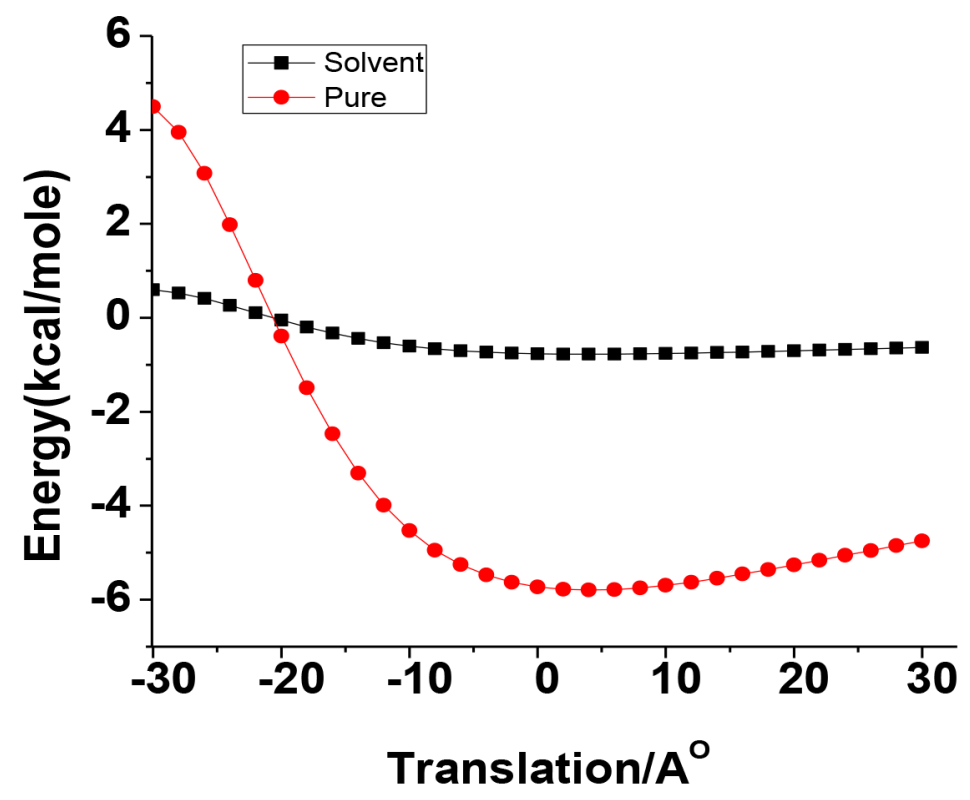

Figure 3: Variation of in-plane interaction energy components with respect to translation along $\mathrm{x}$-axis corresponding to the configuration $\mathrm{y}\left(0^{\circ}\right)$ for pure alkenyl compound and in the solvent (THF).

\section{THERMODYNAMIC APPROACH}

In order to elucidate the results and enhance our understanding of thermodynamic parameters like Helmholtz free energy (A), configurational entropy (S) and internal energy $(\mathrm{U})$ have been estimated. Calculation of these parameters has been useful in understanding the degree of freedom and stability of the given sample and its phase analysis. These parameters during the stacked and in-plane mode of the alkenyl compound in THF medium is presented in Table 1. 
Table 1: Thermodynamic parameters of 4-alkenyl bicyclohexylnitrile in THF medium.

\begin{tabular}{|c|c|c|c|}
\hline \multirow{2}{*}{ Mode of interaction } & \multirow{2}{*}{ Parameter } & \multicolumn{2}{|c|}{ Temperature } \\
\hline & & $300 \mathrm{~K}$ & $364.7 \mathrm{~K}$ \\
\hline \multirow[t]{3}{*}{ Stacking } & $\begin{array}{l}\text { Helmholtz free energy } \\
\left(\mathrm{kcal} \mathrm{mole}^{-1}\right)\end{array}$ & 1.60 & -2.08 \\
\hline & Internal energy $\left(\mathrm{kcal} \mathrm{mole}^{-1}\right)$ & 0.28 & 0.29 \\
\hline & $\begin{array}{l}\text { Entropy } \times 10^{-3} \\
\left(\text { kcal mole } \mathrm{K}^{-1}\right)\end{array}$ & 6.34 & 6.52 \\
\hline \multirow[t]{3}{*}{ In-plane } & $\begin{array}{l}\text { Helmholtz free energy } \\
\left(\mathrm{kcal} \mathrm{mole}^{-1}\right)\end{array}$ & -1.82 & -2.25 \\
\hline & Internal energy $\left(\mathrm{kcal} \mathrm{mole}^{-1}\right)$ & 0.13 & 0.15 \\
\hline & $\begin{array}{l}\text { Entropy } \times 10^{-3} \\
\left(\mathrm{kcal} \text { mole } \mathrm{K}^{-1}\right)\end{array}$ & 6.55 & 6.61 \\
\hline
\end{tabular}

\subsection{Helmholtz Free Energy}

From Table 1, the Helmholtz free energy of the alkenyl compound in THF medium during stacking mode undergoing phase transition at $364.7 \mathrm{~K}$ is found to be $-2.08 \mathrm{kcal} \mathrm{mole}^{-1}$ and $-1.60 \mathrm{kcal} \mathrm{mole}^{-1}$ at room temperature $(300 \mathrm{~K})$. There is a negative increment in the free energy with increase in temperature. This indicates greater stability of phase at the transition temperature. Due to the increase in temperature, a rise in the disorderness causes greater freedom of the molecules. A similar tendency is observed in the in-plane interaction mode interaction, where the value of free energy at the transition temperature $(364.7 \mathrm{~K})$ is $-2.25 \mathrm{kcal} \mathrm{mole}^{-1}$ and at room temperature $(300 \mathrm{~K})$ is $-1.82 \mathrm{kcal} \mathrm{mole}^{-1}$. Albeit similar trend is observed in both the cases, the individual values of free energies at room and transition temperature is found to be prominent during in-plane mode magnitude wise. Therefore, the furnished data suggests that the stability of the phase is higher during the in-plane mode.

\subsection{Internal Energy}

It is well known that the internal energy of a system refers to the total energy of the system, which includes all forms of energies possessed by the atoms and molecules of the system. It not only refers to the motion kinetic energy of molecules inside the system but also provides information about the change in internal configuration and order of the system in terms of energy loss or gain. From the internal energy data, it is observed that there is an increment in the internal energy value in both modes, but the relative increase in the in-plane is 
more than stacking suggesting that internal binding forces required for supporting and maintaining the structural changes in the former are higher as compared to later. This justifies that since stability is more in the in-plane mode, it is the most favoured configuration.

\subsection{Interpretation of Entropy Data}

To explicate the phase analysis of alkenyl compound, we consider the thermodynamic approach in terms of entropy of configurations. Entropy calculation gives additional information on molecular orientation and ease of formation of the liquid crystal phase. Variation of translational entropy during stacking and in-plane interactions at room temperature $(300 \mathrm{~K})$ and transition temperature $(364.7 \mathrm{~K})$ for an alkenyl compound can be seen in Table 1 . The observed value of entropy for stacking interaction at room and transition temperature is $6.34 \times$ $10^{-3} \mathrm{kcal} \mathrm{mole}{ }^{-1} \mathrm{~K}^{-1}$ and $6.52 \times 10^{-3} \mathrm{kcal} \mathrm{mole}^{-1} \mathrm{~K}^{-1}$. Further, during the in-plane mode, the values are $6.55 \times 10^{-3} \mathrm{kcal} \mathrm{mole}^{-1} \mathrm{~K}^{-1}$ and $6.61 \times 10^{-3} \mathrm{kcal} \mathrm{mole}^{-1} \mathrm{~K}^{-1}$, respectively. In both cases, there is an increment in the entropy from room to phase temperature. The relative entropy value in the in-plane mode is lesser than the stacking that reflects that the degree of randomness or disorderness is less in in-plane mode. This causes greater phase stability and order, favouring a liquid crystalline behaviour. Thus, the enumerated points suggest that the in-plane mode of interaction is stable. Hence, the alkenyl compound has been concluded as a nematic liquid crystal.

\section{UV-VISIBLE ABSORPTION SPECTRA}

The spectral data are shown in Table 2, whereas Figure 4 shows the UV-Visible absorption spectra of the alkenyl compound. It is found that alkenyl mesogen in pure form exhibits one absorption peak at $217.58 \mathrm{~nm}$. Further, it exhibits two peaks in the UV region with absorption maxima at $278.51 \mathrm{~nm}$, and one peak in the visible region at $527.54 \mathrm{~nm}$. The UV data indicates a red shift of absorption wavelength that indicates higher UV stability of the compound in THF medium. However, the solvent effect does not show any bandgap variation of the compound. 
Table 2: The absorption bands (AB), extinction coefficients (EC), oscillator strength $(f)$, vertical transition energy $\left(E_{V}\right)$, dipole moment $(\mu), \operatorname{HOMO}(\mathrm{H})$ and LUMO $(\mathrm{L})$ energies, and the bandgap $\left(\mathrm{E}_{\mathrm{g}}=\mathrm{E}_{\mathrm{LUMO}}-\mathrm{E}_{\mathrm{HOMO}}\right)$ of alkenyl compound in pure and in solvent (THF) using CNDO/S method.

\begin{tabular}{lccccc}
\hline & $\mathrm{AB}(\mathrm{nm})$ & $\mathrm{EC}^{*}$ & $f$ & $E_{V}(\mathrm{eV})$ & $\mu(\mathrm{D})$ \\
\hline Pure & $217.58^{*}$ & 0.0004 & 0.0001 & 5.67 & 6.06 \\
$\mathrm{THF}$ & 250.98 & 0.0017 & 0.002 & 4.95 & 7.24 \\
& $278.51^{*}$ & 0.0019 & 0.002 & 4.45 & 7.23 \\
& 527.54 & 0.0005 & 0.0005 & 2.35 & 6.06 \\
\hline
\end{tabular}

Notes: ${ }^{*}=\lambda_{\max }(\mathrm{nm}) ; H=-9.74 \mathrm{eV} ; \mathrm{L}=0.42 \mathrm{eV} ; E_{g}=10.16 \mathrm{eV} ; E C$ unit $=10^{4} \mathrm{dm}^{3} \mathrm{~mol}^{-1} \mathrm{~cm}^{-1}$

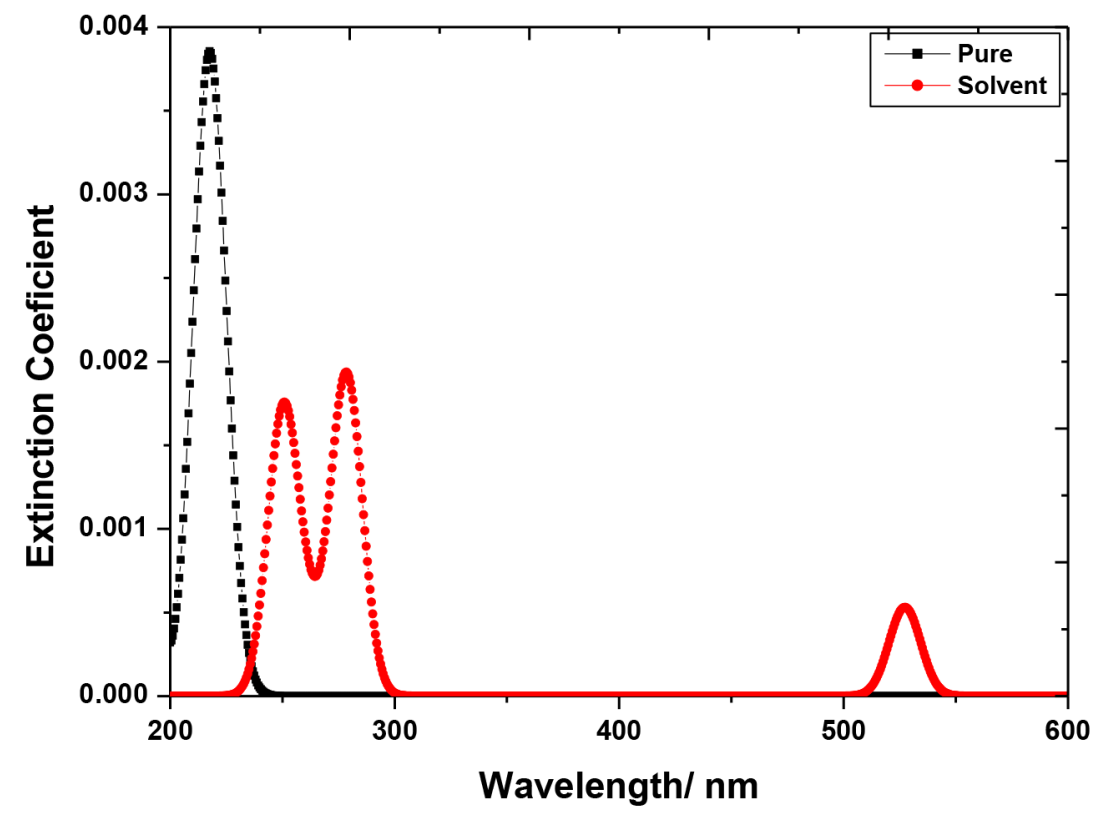

Figure 4: UV-Visible absorption spectra of the alkenyl compound in pure form and in solvent (THF) using CNDO/S method.

\section{CONCLUSION}

From the present approach on the alkenyl compound, a few points may be summarised. At higher temperature in-plane mode of interaction is favoured in terms of increment of internal energy and decrease of entropy and free energy, 
thus suggesting a long rod-like structure oriented along a preferred direction, which favours the nematic phase. Further, since the in-plane mode is favourable after the thermal rise, it indicates that the molecule alignment is unidirectionally stretched along an axis forming a threadlike structure while maintaining the isotropic centre of mass distribution. From this, it is evident that it has ordering (from entropy data) and higher symmetry. The solvent effect causes red-shift of absorption wavelength, thus enhancing the UV stability of the compound.

\section{REFERENCES}

1. Blinov, L. M. (2011). Structure and properties of liquid crystals. New York: Springer.

2. Kundu, P. et al. (2019). Structures and phase transition in a two-dimensional system of Gay-Berne molecules. J. Mol. Liq., 296(1), 111769. https://doi. org/10.1016/j.molliq.2019.111769

3. Das, P. et al. (2019). Solvent polarity and end chain length effects in thermotropic mesophase formation process: Comparative quantum and thermotropic analysis. $J$. Mol. Liq., 288(1), 1-7. https://doi.org/10.1016/j.molliq.2019.111029

4. Prasad, S. \& Ojha, D. P. (2019). Computational study of molecular geometries, and vibrational characteristics of two liquid crystalline disubstituted biphenylcyclohexanes using ab-initio methods. Mol. Cryst. Liq. Cryst., 682(1), 27-43. https://doi.org/10.1080/15421406.2019.1655975

5. Das, P. et al. (2017). Probabilistic and thermodynamic approach on phase behavior of nematic liquid crystals: A comparative study. Mol. Cryst. Liq. Cryst., 652(1), 185-194. https://doi.org/10.1080/15421406.2017.1373009

6. Singh, P. et al. (2019). Omnidirectional reflection band of one-dimensional periodic structure (1DPS) of $\mathrm{Si} / \mathrm{SiO} 2$ with defect mode of nematic liquid crystal (5CB). J. Phys. Sci., 30(3), 117-129, https://doi.org/10.21315/jps2019.30.3.8

7. Ahu, S. et al. (2020). Evaluation of mesomorphic and thermal stabilities for terminal epoxy liquid crystals. J. Mol. Liq., 317(1), 113995. https://doi. org/10.1016/j.molliq.2020.113955

8. Aneela, R. et al. (2012). Role of configurational entropy on ordering and phase organization of nematic liquid crystals - A molecular model. J. Mol. Liq., 166(1), 70-75. https://doi.org/10.1016/j.molliq.2011.12.001

9. Jose, T. J. et al. (2019). Quantum and thermodynamic estimation of mesostate behaviour of alkyl benzoic acids in dielectric medium. Arab. J. Sci. Eng., 44(7), 6601-6608. https://doi.org/10.1007/s13369-019-03791-0

10. Ninham, B. W. (1980). Long-range vs. short-range forces: The present state of play. J. Phys. Chem., 84(12), 1423-1430. https://doi.org/10.1021/j100449a001

11. Lakshmi Praveen, P. \& Ojha, D. P. (2010). Computer simulations of ordering in a nematogen - The role of dielectric medium. Z. Naturforsch., 65(6-7), 555-560. https://doi.org/10.1515/zna-2010-6-711 
12. Kumar, A. et al. (2017). Freezing transitions in a system of two-dimensional octupolar multipoles. Euro. Phys. J. E, 40(9), 80. https://doi.org/10.1140/epje/ i2017-11572-x

13. Klopov, V. I. \& Syrbu, S. A. (2000). Viscosity and statistical thermodynamic properties of 4-alkyloxy-4'-propyloxyazoxybenzenes in the nematic and isotropic liquid states. Th. Found. Chem. Eng., 34, 502-505. https://doi.org/10.1007/ BF02827397

14. Gupta, S. et al. (2001). Structural analysis of mesogenic 4-alkenyl bicyclohexylnitrile. Liq. Cryst., 28, 1309-1313. https://doi. org/10.1080/02678290010025477

15. Pople, J. A. \& Beveridge, D. L. (1970). Approximate molecular orbital theory. New York: McGraw Hill.

16. Pullmann, B. (ed.). (1978). Intermolecular interactions: From diatomic to biopolymers. New York: John Wiley:

17. Lakshmi Praveen, P. et al. (2011). Role of molecular rigidity on phase organization of a smectic liquid crystal-A theoretical model. Mat. Chem. Phys., 126, 248-252. https://doi.org/10.1016/j.matchemphys.2010.11.031

18. Kitaygorodsky, A. I. (1961). The interaction curve of non-bonded carbon and hydrogen atoms and its application. Tetrahedr., 14 (3-4), 230-236. https://doi. org/10.1016/S0040-4020(01)92172-6

19. Ojha, D. P. (2001). Nematogenic behaviour of a cyano compound using quantum mechanics and computer simulation. Z. Naturforsch., 56a(3-4), 319-325. https:// doi.org/10.1515/zna-2001-0315

20. Hirschfelder, J. O., Curtiss, C. F. \& Bird, R. B. (1967). Molecular theory of gases and liquids. New York: John Wiley \& Sons.

21. Jose, T. J. et al. (2018). Theoretical model of a nematogen: Estimation of phase stability, absorption, electrochemical, and non-linear optical properties. Acta Phys. Pol. A, 134(2), 512-518. https://doi.org/10.12693/APhysPolA.134.512

22. Ghosh, S. et al. (2017). Modeling optical spectra of large organic systems using real-time propagation of semiempirical effective Hamiltonians. J. Chem. Theory Comput., 13(9), 4410-4420. https://doi.org/10.1021/acs.jctc.7b00618

23. Lakshmi Praveen, P. et al. (2014). Photo sensitivity, substituent, and solvent induced shifts in UV-visible absorption bands of naphthyl-ester liquid crystals: A comparative theoretical approach. Liq. Cryst., 41(6) 872-882. https://doi.org/10.1 080/02678292.2014.882423

24. Gayatri, V. et al. (2010). Computer simulation of molecular organization in a nematogen. The role of thermodynamic parameters. Russ. J. Gen. Chem., 80(10), 2048-2053. https://doi.org/10.1134/S1070363210100270

25. Ojha, D. P. et al. (2013). Theoretical study on liquid crystal cyanobiphenyls: Phase stability and phase behaviour. J. Phys. Chem. Solids, 74(11), 1653-1659. https:// doi.org/10.1016/j.jpcs.2013.07.001 